\title{
Management of Respiratory Complications after Maxillofacial Surgery on Board Ship
}

\author{
DrKDThomson \\ FFARCS \\ Consultant Anaesthetist
}

East Berkshire

SUMMARY: An eight year old girl who probably inhaled gastric contents during surgery for cleft palate repair was ventilated for 10 days in the post-operative recovery area on board the Merey Ship Anastasis with pulse oximetry as the only monitor of oxygenation. She walked off the ship on the seventeenth day.

\section{Case Report}

A previously fit eight year old Ghanaian girl was scheduled for elective pharyngoplasty, palatoplasty and cleft lip repair (Fig 1). Her pre-operative haemoglobin

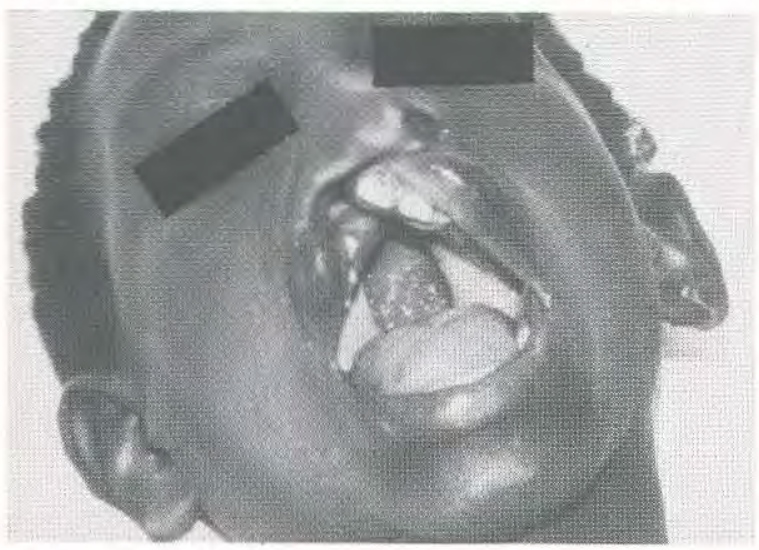

Fig 1. Pre-operative photograph.

was $10.4 \mathrm{~g} / \mathrm{dl}$, white cell count $8.7 \times 10^{9 / 1}$, and platelets $329 \times 10^{9} / 1$. Serology was negative for human immunodeficiency virus (HIV), rapid plasma reagin (RPR), sickledex and malaria.

After premedication with oral diazepam $2 \mathrm{mg} 90 \mathrm{mins}$ pre-operatively inhalation induction was performed using halothane and nitrous oxide in oxygen. She was then intubated with a $6.0 \mathrm{~mm}$ RAE orotracheal uncuffed tube; an appropriate leak was obtained but the tube was too long when the surgeon's gag was positioned, so it was shortened by cutting about $1.5 \mathrm{cms}$ off the distal end and reinserted.

During two hours of surgery, anaesthesia was maintained by spontaneous respiration of halothane $1-2 \%$ in oxygen supplemented by intermittent intravenous bolus doses of pethidine, but the oxygen saturation ( $\mathrm{Sa02}$ ) gradually began to decrease; breath sounds were heard on auscultation during assisted ventilation, suggesting the tube was correctly positioned, but an increased gas flow from that normally used of one litre per minute, via an Ohio circle system, to keep the reservoir bag suitably

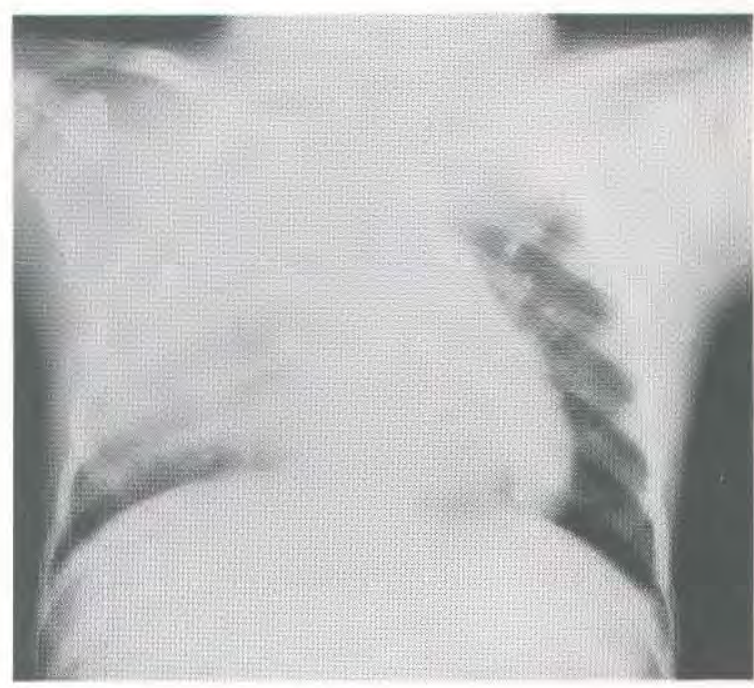

Fig 2. Post-operative CXR.

distended should perhaps have alerted the anaesthetist to the possibility of a wrongly placed tube. At the end of the operation the $\mathrm{SaO} 2$ was only $83 \%$ on $100 \%$ oxygen and after removal of the blood stained throat pack the tracheal tube was found to be out of the larynx, spontaneous ventilation was not satisfactory and a chest Xray (Fig 2) showed diffuse shadowing throughout most of the right lung and similar shadowing in the left upper zone. The appearance was in keeping with an aspiration pneumonitis as there was no evidence of volume loss or mediastinal shift and the upper lobes were more affected which is consistent with aspiration in the supine position.

Ghana has no facilities for long term ventilation, so the patient was managed in the post-operative recovery area on the ship; she was connected to a Bains type breathing system, the proximal end of which was attached to the fresh gas outlet of an anaesthetic machine from which changes in the inspired oxygen concentration could be obtained by appropriate alterations to the oxygen flow rate. Ventilation was achieved using room air as the driving gas by attaching an old electrically 
driven Bennett to the expiratory limb of the Bains system via the reservoir bag connection. The expiratory tubing from the ventilator was placed in a flask of water to generate positive end expiratory pressure (PEEP) the magnitude of which could be altered by varying the length of tubing positioned vertically below the water level. The initial introduction of 5-6 cms of PEEP increased the Sa02 dramatically from $82 \%$ to $92 \%$. Sa02, blood pressure, ECG, urine output and nasogastric aspirate were continuously monitored, but there was no facility on board to measure blood gases, inspired oxygen concentration or end tidal carbon dioxide. Sedation when required was with intravenous bolus doses of midazolam fentanyl and vecuronium. Antacid chemoprophylaxis was initially with intravenous ranitidine and subsequently with sucralfate via a nasogastric tube.

The following day her white cell count rose to $26.4 \mathrm{x}$ $109 / 1$ and gram negative cocci and rods were seen on sputum microscopy. She was started on intravenous ceftizoxime. Her airway pressure was now $40 \mathrm{cms}$ of water. By day 4 her haemoglobin and platelets had decreased to $8.0 \mathrm{~g} / \mathrm{dl}$ and $81.0 \times 10^{9 / 1}$ respectively and the white cell count to $12.0 \times 10 \% / 1$.

For ease of management, particularly to facilitate suction and weaning, a tracheostomy was performed on day 5 and an appropriate sized Portex uncuffed tube inserted. Her condition gradually began to improve and weaning off the ventilator commenced on day 6 . By day 7 she breathed for some hours on a continuous positive airway pressure (CPAP) circuit, but on the afternoon of day 8 her condition deteriorated dramatically, ventilation became difficult and the saturation fell to $60 \%$ for nearly 20 minutes. An an X-ray was considered to be essential, to eliminate treatable chest pathology (e.g. pneumothorax), she was carried along the ship's corridor to the X-ray machine and the subsequent chest X-ray (Fig 3) showed that the tip of the tracheostomy tube was almost touching the carina; it also showed resolving right sided consolidation with some residual consolidation

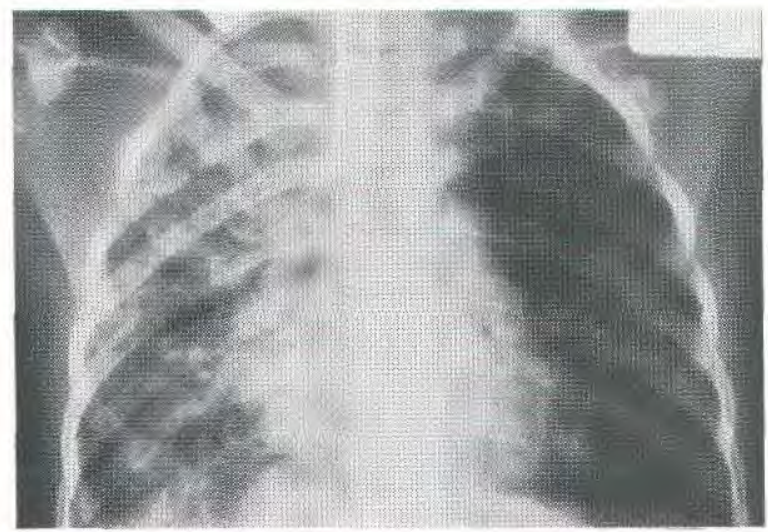

Fig 3. CXR - day 8. also in the left mid and lower zones. The acute episo was probably due to secretions transiently blocking or of the main bronchi so the tracheostomy tube w及 withdrawn one centimetre and kept in position with piece of polystyrene.

The next day, after 36 hours of full ventilation 응 sputum sample culture showed a profuse growth of gran negative rods which were sensitive to gentamicin at? ticarcillin. As ticarcillin was not available, she wo started on appropriate doses of intravenous gentamic and piperacillin, also quinine sulphate via th nasogastric tube for malaria which had been demo strated on a blood film. A $250 \mathrm{ml}$ blood transfusion wâ also given.

On the morning of day 10 , her chest sounded drama $\vec{P}$ ically better on auscultation. Her oxygen saturation stayed at $98 \%$ and inspiratory pressure had decreased $\mathscr{H}^{\circ}$ $15 \mathrm{cms}$ of water. She was taken off the ventilator a a t? allowed to breathe spontaneously on a continuous po tive airways pressure (CPAP) circuit as illustrated Hinds (1). She was gradually weaned off the CPAD during the following 48 hours and attached to a 'torpe d' tube' $(60 \mathrm{ml}$ syringe drilled with the appropriate size of hole) connected to oxygen tubing. The torpedo tube alst acted as a convenient trap for the sputum which was tof being coughed up in copious amounts as her secon pneumonia rapidly resolved.

Her condition continued to improve; on day $1 \frac{1}{\mathrm{D}_{\mathrm{S}}} \overrightarrow{\mathrm{D}}_{\mathrm{e}}$ maintained an adequate saturation on room air; the day the tracheostomy tube was removed and on das 8 she walked off the ship, the day before the Ana요요 sailed on schedule for Europe.

\section{Discussion}

This girl's respiratory problems occurred because के the unrecognised fact that the tracheal tube had becon displaced, probably either at re-intubation or duris positioning of the head by the surgeon after the tube hag been shortened. The problem of the recommended si by age $(4.0+$ age $/ 4)$ of pre-formed oral tubes being to long, has been previously noticed by Lowson (2), wh $\bar{B}_{\mathrm{e}}$ working in Nepal; he suggested that it was a result poor nutrition or familial short stature. However Blaks and Mackersie (3), in a study at Great Ormond Str $\widehat{\oplus}_{t}$ Hospital found that accidental bronchial intubati occurred in 20 per cent of their series. It should also be noted that in 1986, Mallinckrodt shortened the paediatric size RAE tubes by up to $1.5 \mathrm{cms}$; as all tie tubes on the ship had been donated free of charge there a possibility that they were manufactured before 1986.0

After considering the clinical chain of events and the $\mathrm{X}$-ray findings, the most likely diagnosis was acute piration pneumonitis followed by secondary pneum nia.

Aspiration pneumonia has a significant mortality ev긍 in the best equipped intensive care units in the develond्ञ world; but in spite of the lack of a modern ventilator agd 
'essential' equipment to monitor blood gases and inspired oxygen concentration a young girl was kept alive until her life threatening pathology resolved, by dedicated nurses giving all prescribed drugs, provision of regular suction, physiotherapy and monitoring 24 hours a day for two weeks and by effective use of available equipment including a flask of water as a PEEP valve, a CPAP circuit, an early tracheostomy to facilitate suction and a pulse oximeter.

The pulse oximeter proved its value as an exceptional monitor, by both providing an early warning signal of developing problems and also rapidly indicating the effect on oxygen saturation of varying such parameters as inspired oxygen flow rate, tidal volume, respiratory rate and the amount of PEEP.

It would be difficult also to forget the unquantifiable effect of the 450 'volunteers' on the Anastasis, all supporting the efforts of the medical team and 'willing' this young girl to survive and make a full recovery.

\section{REFERENCES}

1. Hinds C J. Intensive Care a Concise Textbook, Balliere Tindall, 1987: 245.

2. Lowson S. Anaesthesia and ENT camps in Nepal. Today's Anaesthetist 1991; 6: 45-46.

3. Black A E, Mackersie A M. Accidental Bronchial intubation with RAE tubes. Anaesthesia 1991; 46: $42-43$. 